\title{
Stent versus bypass: The reasons and risk factors for early readmission to hospital after myocardial revascularization
}

\author{
Murat Sargin, ${ }^{1}$ Mustafa Adem Tatlisu, ${ }^{2}$ Muge Tasdemir Mete, ${ }^{1}$ Nehir Selcuk, ${ }^{1}$ Sevinc Bayer, ${ }^{1}$ \\ Serdar Akansel, ${ }^{1}$ Serap Aykut Aka, ${ }^{1}$ Mehmet Eren ${ }^{2}$ \\ ${ }^{1}$ Siyami Ersek Thoracic and Cardiovascular Surgery Center Cardiovascular Surgery Clinic, Istanbul, Turkey \\ ${ }^{2}$ Siyami Ersek Thoracic and Cardiovascular Surgery Center Cardiology Clinic, Istanbul, Turkey
}

\begin{abstract}
OBJECTIVE: Though 30-day rates of readmission for coronary artery bypass graft (CABG) surgery and percutaneous coronary intervention (PCI) remain high, readmission rates and associated risk factors have not been well examined. The purpose of the present study was to determine the risk factors for and rates of readmission and to compare two revascularization methods on that basis.
\end{abstract}

METHODS: The study included 2664 consecutive patients who underwent coronary revascularization either with CABG surgery or PCI. The study was performed retrospectively and a wide variety of risk factors related to readmission were selected for analysis, including demographic data, preoperative risk factors and postoperative complications.

RESULTS: From the CABG group (Group 1, $\mathrm{n}=1103$ ), 18.3\% were readmitted, as were $15.2 \%$ of the PCI group (Group 2, $\mathrm{n}=1561$ ). In multivariate analysis, age, gender, left ventricular ejection fraction (LVEF), chronic obstructive pulmonary disease (COPD), diabetes mellitus (DM), length of stay (>10 days), body mass index (BMI), and creatinine level on admission were associated with early readmission for group 1 (Table 3). In group 2, age, gender, LVEF, DM, length of stay (>10 days), and creatinine level on admission were associated with early readmission.

CONCLUSION: When two methods of revascularization were compared, rates of readmission were found to be similar. Patients with cited risk factors are prone to readmission in the first 30 days, so extra precautions should be taken at discharge. Neither method can be concluded to be superior with regard to readmission rates.

Keywords: Aorta-coronary bypass; percutaneous coronary intervention; readmission.

Received: March 14, 2016 Accepted: May 04, 2016 Online: May 15, 2016

Correspondence: Dr. Murat Sargin. Tibbiye Caddesi, 34000 Uskudar, Istanbul, Turkey

Tel: +902163379920 e-mail: muratsargin@gmail.com

(c) Copyright 2016 by Istanbul Northern Anatolian Association of Public Hospitals - Available online at www.kuzeyklinikleri.com 
$T$ he incidence of coronary artery disease has increased, yet despite new effective medical therapies, new drug-eluted stents, and novel surgical techniques developed, the early period following revascularization after discharge is prone to many problems. Risk factors for morbidity and mortality related to coronary artery bypass graft (CABG) surgery and percutaneous coronary intervention (PCI) have been extensively studied. The 30-day rate of readmission for both revascularization methods remains high, despite decreases in mortality $[1,2,3]$. However, readmission rates and associated risk factors have not been well examined. In particular, the two methods have not been compared with respect to early readmission.

The primary objective of the present study was to determine the risk factors for and rates of readmission in the first 30 days after surgical or percutaneous revascularization. The secondary goal was to use the data to compare the two surgical methods.

\section{MATERIALS AND METHODS}

The study included 2664 consecutive patients with coronary artery disease who underwent revascularization at the hospital either with CABG surgery or PCI between January 1 and September 1, 2013. The study was performed retrospectively and approved by the scientific board of the hospital. Of these patients, 1561 had PCI, and 1103 had CABG surgery. The patients included underwent only either CABG or PCI. Patients who died while in hospital and surviving patients who had not been discharged by postoperative day 30 were excluded. Patients who received concomitant surgeries or procedures were also excluded. In this study cohort, the goal was to identify patients who were readmitted within 30 days after discharge for a variety of complications of CABG surgery or PCI and to compare them to patients who were not readmitted within 30 days of discharge. A wide variety of potential risk factors related to readmission within 30 days, such as demographic data, preoperative risk factors and postoperative complications were selected for analysis, in addition to other variables. These included demographic data such as age and gender, comorbidities (diabetes mellitus [DM], chronic obstructive pulmonary disease [COPD]), previous revascularization (PCI or CABG), length of hospital stay (1-10 days, more than 10 days) postoperative complications such as bleeding revision, intra-aortic balloon pump requirement, major complications such as sepsis, gastrointestinal bleeding, renal failure, or respiratory failure. The rates of readmission, hospitalization and risk factors were analyzed.

\section{Statistical analysis}

All statistical analyses were performed using SPSS software (version 21.0; SPSS Inc., Chicago, IL, USA) and $p$ value $<0.05$ was considered statistically significant. Categorical variables are expressed as $n$ (\%), and continuous variables are expressed as mean $+\mathrm{SD}$. Forward stepwise multivariate logistic regression models were created to identify the independent predictors of 30-day readmission to emergency room (ER) after myocardial revascularization. Variables with $\mathrm{p}$ value $<0.05$ in univariate analysis were included in the multivariate model. To estimate the impact of demographic, clinical and angiographic variables on occurrence of any cause for ER services, we performed multiple logistic regression analysis, including all variables with a univariate relationship $(\mathrm{p}<0.10)$.

\section{RESULTS}

The study included 2664 consecutive patients with coronary artery disease undergoing coronary revascularization, and these patients were divided into two groups: CABG group (Group 1, $\mathrm{n}=1103$ ) and PCI group (Group 2, n=1561). A total of 440 (16.5\%) of 2664 patients were readmitted within 30 days of discharge following revascularization. Of the CABG group, $18.3 \%(n=202)$ were readmitted, as were $15.2 \%(n=238)$ of the PCI group. The number of patients and their baseline demographic and clinical data are presented in Figure 1 and Table 1.

Cardiac care prompted the readmission of $33.6 \%(n=148)$, while $66.4 \%(n=292)$ of patients were admitted for non-cardiac reasons (diabetes-associated problems, respiratory infections, non-specific). The most frequent 6 reasons for readmission 


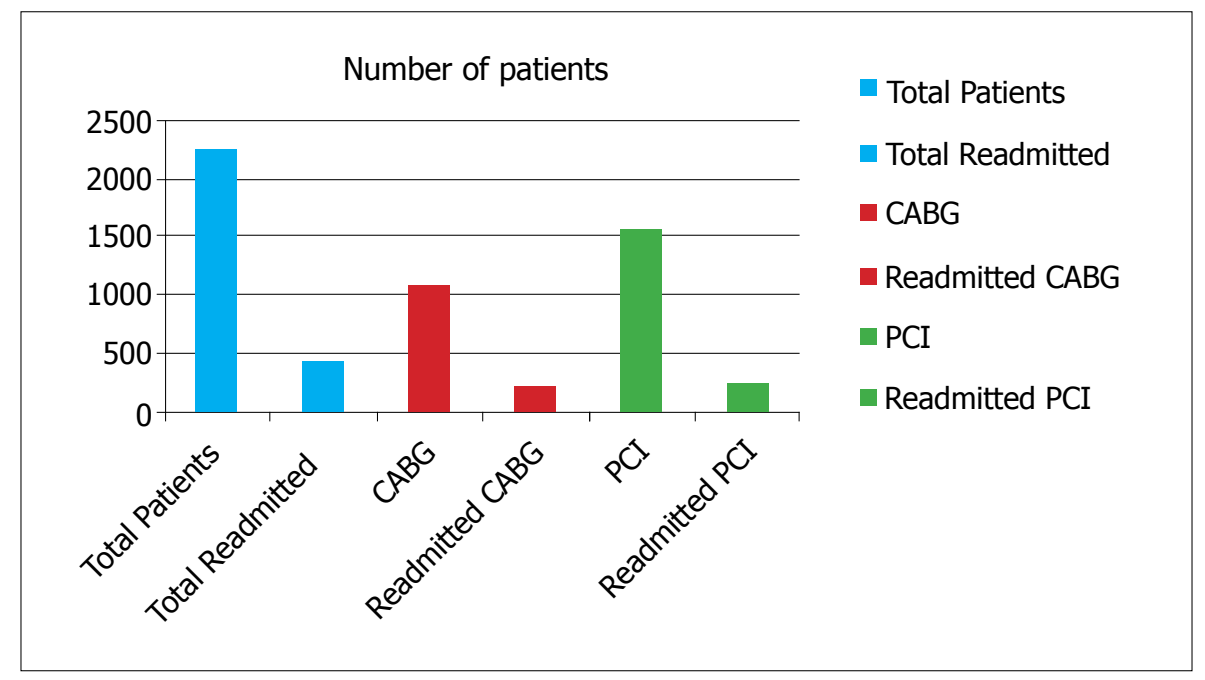

FIGURE 1. Number of patients retrospectively studied in the analysis. CABG: Coronary artery bypass graft surgery; PCI: Percutaneous coronary intervention.

for whole patient group were dysrhythmia (15.7\%), nonspecific causes $(15.1 \%)$, heart failure $(10.2 \%)$, respiratory problems $(9.8 \%)$, infection $(7.2 \%)$, and vascular access site complications (6.8\%). Principal reasons for readmission for Group 1 and Group 2 are presented in Table 2 .
Of the readmissions, $15.38 \%$ of CABG group $(n=32)$ and $17.2 \%$ of PCI group $(n=41)$ were rehospitalized. The rehospitalization rate for the whole cohort was $2.7 \%$; that is, $2.9 \%$ of all CABG patients and $2.6 \%$ of all PCI patients. The approach to treatment of complications was standard hospital

TABLE 1. Comparison of demographic and clinical features of patients in Group 1 and in Group 2 at presentation $(n=2664)$

\begin{tabular}{lccc} 
Variables & Group 1 $(\mathrm{n}=1103)$ & Group 2 $(\mathrm{n}=1561)$ & $\mathrm{p}$ \\
\hline Age (years) & $65.1 \pm 10.6$ & $56.5 \pm 10.6$ & 0.03 \\
Male & $609(55.3 \%)$ & $914(58.6 \%)$ & 0.21 \\
Hypertension & $756(68.5 \%)$ & $566(36.2 \%)$ & 0.02 \\
DM & $286(25.9 \%)$ & $276(17.7 \%)$ & 0.18 \\
COPD & $203(18.4 \%)$ & $217(13.9 \%)$ & 0.22 \\
Hyperlipidemia & $330(29.9 \%)$ & $374(23.9 \%)$ & 0.35 \\
LDL-cholesterol (mg/dL) & $111.2 \pm 32.2$ & $120.1 \pm 33.1$ & 0.06 \\
History of smoking & $551(49.9 \%)$ & $858(54.9 \%)$ & 0.23 \\
Previous revascularization & $117(10.6 \%)$ & $219(14.02 \%)$ & 0.04 \\
EF (\%) (on readmission) & $37.5 \pm 10.5$ & $47.6 \pm 8.6$ & 0.01 \\
Creatinine (mg/dL) (on readmission) & $1.34 \pm 1.02$ & $0.88 \pm 0.25$ & 0.01 \\
BMI>30 & $91(8.3 \%)$ & - & \\
Length of stay>10 days & $103(9.3 \%)$ & $96(6.14 \%)$ & 0.07 \\
\hline
\end{tabular}

BMI: Body mass index; COPD: Chronic obstructive pulmonary disease; DM: Diabetes mellitus; EF: Ejection fraction.

* Parametric variables are reported in mean \pm SD or median (interquantile range); categorical variables are reported in number (percentage). Hypertension: Patients using medication for hypertension; DM: Patients using oral antidiabetics or insulin; Smoking: Declaration of smoking, regardless of packs/years; COPD: Use of COPD medications before or after revascularization. 


\begin{tabular}{lcc}
\multicolumn{4}{l}{ TABLE 2. Principal causes of readmission for both Group 1 and Group 2} \\
\hline Principal Diagnosis & Group 1 (\%) & Group 2 (\%) \\
\hline \multicolumn{1}{c}{ Cardiac } & 33.1 & 34.3 \\
Myocardial infarction/Ischemia & 2.9 & 3.3 \\
Angina pain/Chest pain & 3.8 & 4.2 \\
Heart failure & 8.9 & 9.4 \\
Dysrhythmia & 13.2 & 17.4 \\
Postcardiotomy syndrome & 4.3 & - \\
$\quad$ Non-Cardiac & 66.9 & 65.7 \\
Incisional infections & 16.9 & - \\
Respiratory & 14.5 & 10.2 \\
Renal & 2.3 & 3.4 \\
Vascular access site complication & - & 12.3 \\
Other & 19.8 & 23.1 \\
Nonspecific & 13.4 & 16.7 \\
& $\% 100$ & $\% 100$ \\
\hline
\end{tabular}

BMI: Body mass index; COPD: Chronic obstructive pulmonary disease; DM: Diabetes mellitus;

EF: Ejection fraction.

policy for surgical and cardiology teams. Reasons for rehospitalization in the CABG group were primarily deep sternal infection, myocardial ischemia/ infarction, and pleural effusion. For the PCI group, rehospitalization was mainly the result of myocardial ischemia/infarction, arrhythmia, and vascular access site complications. Eight (1.8\% of readmissions) of the readmitted patients underwent coro-

TABLE 3. Univariate and multivariate analysis of the predictors for early readmission in Group 1 population

\begin{tabular}{|c|c|c|c|c|}
\hline & \multicolumn{2}{|c|}{ Univariate Analysis } & \multicolumn{2}{|c|}{ Multivariate Analysis * } \\
\hline & OR $(95 \% \mathrm{CI})$ & $\mathrm{p}$ & OR $(95 \% \mathrm{CI})$ & $p$ \\
\hline Age & $1.5(1.33-1.65)$ & 0.01 & $1.18(1.0-1.32)$ & 0.02 \\
\hline Female gender & $1.3(1.26-1.45)$ & 0.01 & $1.22(1.12-1.32)$ & 0.01 \\
\hline Smoking & $1.56(0.6-4.7)$ & 0.34 & - & - \\
\hline Left ventricular EF & $0.7(0.58-1.02)$ & 0.01 & $0.82(0.61-0.99)$ & 0.03 \\
\hline Hypertension & $1.8(0.9-4.1)$ & 0.22 & - & - \\
\hline DM & $1.9(1.53-2.2)$ & 0.01 & $1.4(1.3-1.66)$ & 0.02 \\
\hline COPD & $1.3(1.08-1.54)$ & 0.01 & $1.09(0.96-1.28)$ & 0.04 \\
\hline Creatinine & $1.42(1.3-1.68)$ & 0.01 & $1.16(0.92 .-1.32)$ & 0.03 \\
\hline BMI & $1.5(1.1-1.78)$ & 0.01 & $1.18(0.92-1.34)$ & 0.03 \\
\hline Previous revascularization & $1.8(1.2-3.4)$ & 0.03 & $1.22(0.9-2.34)$ & 0.12 \\
\hline Length of stay (>10 days) & $1.9(1.43-2.7)$ & 0.01 & $1.3(0.96-1.41)$ & 0.02 \\
\hline
\end{tabular}

BMI: Body mass index; CI: Confidence interval; COPD: Chronic obstructive pulmonary disease; DM: Diabetes mellitus; EF: Ejection fraction; OR: Odds ratio. *Variables with a p value $<0.05$ in univariate analysis were included in the multivariate model. +Each parameter was studied separately using multiple regression analysis. BMI: Body mass index; COPD: Chronic obstructive pulmonary disease; DM: Diabetes mellitus; EF: Ejection fraction. 


\begin{tabular}{|c|c|c|c|c|}
\hline & \multicolumn{2}{|c|}{ Univariate Analysis } & \multicolumn{2}{|c|}{ Multivariate Analysis * } \\
\hline & OR $(95 \% \mathrm{CI})$ & $\mathrm{p}$ & OR $(95 \% \mathrm{CI})$ & $\mathrm{p}$ \\
\hline Age & $1.1(1.03-1.15)$ & 0.01 & $1.085(1.042-1.132)$ & 0.01 \\
\hline Female gender & $1.6(1.3-1.7)$ & 0.01 & $1.2(1.08-1.34)$ & 0.04 \\
\hline Smoking & $1.6(0.82-4.94)$ & 0.19 & - & - \\
\hline Left ventricular EF & $0.91(0.88-0.94)$ & 0.01 & $0.95(0.91-0.99)$ & 0.01 \\
\hline Hypertension & $1.64(0.81-3.32)$ & 0.16 & - & - \\
\hline DM & $1.64(1.43-1.72)$ & 0.01 & $1.34(1.24-1.46)$ & 0.01 \\
\hline COPD & $1.1(0.9-1.58)$ & 0.02 & $1.01(0.6-1.36)$ & 0.12 \\
\hline Creatinine & $1.59(1.32-1.74)$ & 0.01 & $1.26(1.13-1.42)$ & 0.01 \\
\hline Hemoglobin & $0.7(0.32-2.92)$ & 0.21 & - & - \\
\hline Previous revascularization & $1.6(1.1-4.62)$ & 0.04 & $1.1(0.92-2.6)$ & 0.16 \\
\hline Length of stay ( $>10$ days) & $1.8(1.62-2.3)$ & 0.01 & $1.2(0.9-1.43)$ & 0.03 \\
\hline
\end{tabular}

CI: Confidence interval COPD: Chronic obstructive pulmonary disease; DM: Diabetes mellitus; EF: Ejection fraction; OR: Odds ratio. *Variables with a $\mathrm{p}$ value $<0.05$ in univariate analysis were included in the multivariate model. + Each parameter was studied separately using multiple regression analysis.

nary angiography. Three of the readmitted patients had another PCI, and 4 patients died (2 CABG, 2 PCI).

The univariate and multivariate logistic regression analysis for predictors of early readmission in both Group 1 and Group 2 are presented in Table 3 and Table 4, respectively. Variables with $p$ val- ue $<0.05$ in univariate analysis were included in the multivariate model.

In multivariate analysis, age, gender, LVEF, COPD, DM, length of stay ( $>10$ days), BMI, and creatinine level on admission $(\mathrm{p}<0.05$ for each $\mathrm{pa}$ rameter) were associated with early readmission for Group 1 (Table 3). In Group 2, age, gender, LVEF,

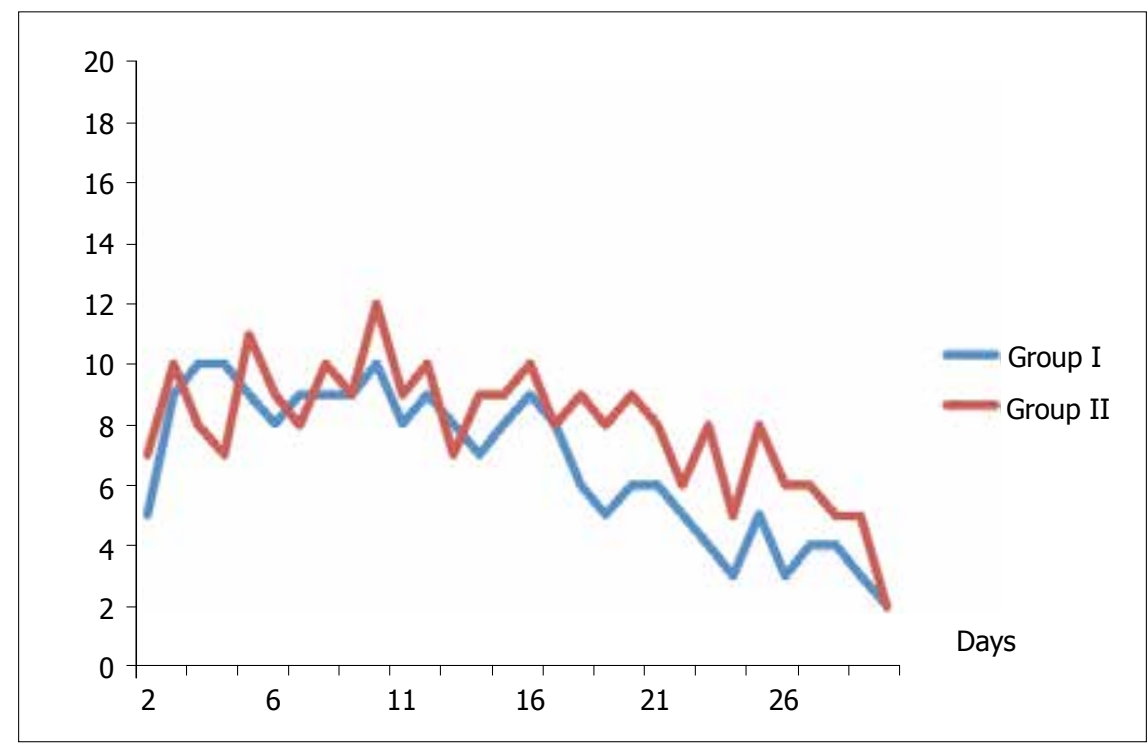

FIGURE 2. Days/number of readmissions. 
DM, length of stay ( $>10$ days), and creatinine level on admission $(p<0.05$ for each parameter) were associated with early readmission (Table 4).

\section{DISCUSSION}

Although long-term results of revascularization methods have been extensively studied, knowledge of early readmission rates and causes is still limited [1-4]. The main objectives of the present study were to document the extent of 30-day readmissions after CABG surgery and PCI, to identify reasons for those readmissions, and to determine independent predictors of readmission for patients who underwent PCI and CABG surgery.

The study results indicated that $16.5 \%$ of all patients, $18.3 \%$ of CABG group, and $15.2 \%$ of PCI group were readmitted within 30 days. The need for cardiac care led to readmission within 30 days of $6.1 \%$ of CABG patients and $5.3 \%$ of PCI patients. The findings were fairly similar to those of other studies.

Hannan et al. studied 33936 patients who underwent $C A B G$ surgery and found that the most common reasons for readmission were post-operative infection $(16.9 \%)$, heart failure $(12.8 \%)$, and "other complications of surgical and medical care" (9.8\%). Increased age, female sex, higher BMI, presence of more than 1 comorbidity, use of saphenous vein grafts, and longer lengths of stay were all associated with higher rates of readmission. The correlation between risk-adjusted 30-day readmission rate of hospitals and risk-adjusted in-hospital/30-day mortality rate was $0.32(\mathrm{p}=0.047)$. The range in the readmission rate across hospitals was $8.3 \%$ to $21.1 \%[5,6]$.

In another study, Hannan et al. retrospectively analyzed 30-day readmissions after PCI using statewide PCI registry to identify $40093 \mathrm{New}$ York State patients who underwent PCI. A total of $15.6 \%$ of all PCI patients were readmitted within 30 days, and most common reasons for readmission were chronic ischemic heart disease (22.5\%), chest pain $(10.8 \%)$, and heart failure (8.2\%). A total of 2015 patients (32.2\% of readmissions) underwent a repeat PCI. Age over 65, female gender, low ejection fraction (EF), COPD, DM, renal failure, and longer stay in hospital after PCI were found to be risk factors [7].

Khawaja et al. reported in a study of 30-day readmission rates after PCI (15498) that overall, 9.4\% of PCI patients $(n=1459)$ were readmitted, and $0.68 \%(n=106)$ died within 30 days after discharge. After multivariate analysis, female sex, education level below high school, unstable angina, cerebrovascular accident or transient ischemic attack, moderate to severe renal disease, COPD, peptic ulcer disease, metastatic cancer, and a length of stay of more than 3 days were associated with an increased risk of 30-day readmission after PCI. Thirty-day readmission after PCI was associated with a higher risk of 1-year mortality (adjusted hazard ratio, 1.38; 95\% confidence interval, 1.08-1.75; $\mathrm{p}=.009$ ) [8].

In the present study, BMI data for the $\mathrm{CABG}$ group were determined to be a risk factor for readmission, usually related to incisional infection. For the PCI group, BMI data were not available for all patients; therefore, it was not included in the analysis. DM, female gender, increased age, congestive heart failure, increased creatinine level on admission, and longer lengths of stay were defined as risk factors for readmission after PCI.

Gender has become an important consideration in revascularization. Female patients are known to have greater rates of morbidity and mortality [1$4]$. Some studies have shown that women were readmitted more than twice as often as men within 30 days of CABG surgery $[4-7,9,10]$. The results of the present study also indicate that significantly more women were readmitted to hospital than men.

DM is a comorbidity that almost all studies define as a risk factor for any cardiac event $[4-8,11]$. Similarly, we found diabetes to be a risk factor for readmission. Diabetic female patients, regardless of which procedure was performed, were readmitted to the hospital more than any other patient group.

The secondary outcome of the study was a comparison of the two groups of revascularization patients in terms of readmission. Diabetes, female gender, obesity and older age were found to be risk factors for readmission for patients of both procedures. In the CABG group, females, diabetics and 
obese patients were readmitted to ER primarily due to infections; however, in the PCI group, the reasons were mostly non-cardiac. Multiple comorbidities, low EF, and emergency revascularization were other common risk factors for cardiac readmissions. Effects of cardiopulmonary bypass, general anesthesia and sternotomy may be primary causes of problems that lead to readmission. Similarity of risk factors for readmission in PCI and CABG groups is evidence that the surgery itself is not the only cause.

Subgroups according to age (30-50 years, 5070 years, over 70 years) were analyzed. In the PCI group, readmission was more frequent in 30-50 age group. Multivariate analysis did not reveal younger age as a risk factor for readmission. Identification of younger age as a risk factor for the PCI group needs further analysis. In our young PCI patient cohort, the most frequent reason for readmission was chest pain without electrocardiography findings. These patients were mostly diabetic. History of smoking and dyslipidemia were higher in these patients and they were often multivessel atherosclerotic patients. We believe these patients had widespread atherosclerosis aside from target vessel PCI, and these patients were more prone to be readmitted to ER for cardiac reasons.

The distribution of number of patients who were readmitted in first 30 days is shown in Figure 2. Readmission rates were higher in the first 10 days than for the remainder of the 30-day period. This finding was similar to those in literature $[4-8,11]$.

\section{CONCLUSION}

Readmission to hospital in the first 30 days after discharge is a common problem. Although we identified a number of important predictors for readmission, many variables associated with readmission remain unexplained. Future research will be needed to better understand why many patients require readmission after revascularization.

When two methods of revascularization were compared, rates of readmission were found to be similar between groups. Neither method can be concluded to be superior with regard to readmission rates.
Conflict of Interest: No conflict of interest was declared by the authors.

Financial Disclosure: The authors declared that this study has received no financial support.

Authorship contributions: Concept - M.S., M.A.T.; Design M.S., M.A.T.; Supervision - S.A.A., M.E.; Materials - M.S., M.A.T, M.E.T.; Data collection and processing - N.S., S.B., S.A.; Analysis and interpretation - M.S., M.A.T., M.E.T., N.S.; Literature Search - S.B., S.A.; Writing - M.S., M.A.T.; Critical Review S.A.A., M.E.

\section{REFERENCES}

1. Stanton BA, Jenkins CD, Goldstein RL, Vander Salm TJ, Klein MD, Aucoin RA. Hospital readmissions among survivors six months after myocardial revascularization. JAMA 1985;253:3568-73. Crossref

2. Kiefe C. Predicting rehospitalization after bypass surgery: can we do it? Should we care? Med Care 1999;37:621-4. Crossref

3. Stewart RD, Campos CT, Jennings B, Lollis SS, Levitsky S, Lahey SJ. Predictors of 30-day hospital readmission after coronary artery bypass. Ann Thorac Surg 2000;70:169-74. Crossref

4. Curtis JP, Schreiner G, Wang Y, Chen J, Spertus JA, Rumsfeld JS, et al. All-cause readmission and repeat revascularization after percutaneous coronary intervention in a cohort of medicare patients. J Am Coll Cardiol 2009;54:903-7. Crossref

5. Hannan EL, Racz MJ, Walford G, Ryan TJ, Isom OW, Bennett E, et al. Predictors of readmission for complications of coronary artery bypass graft surgery. JAMA 2003;290:773-80. Crossref

6. Hannan EL, Zhong Y, Lahey SJ, Culliford AT, Gold JP, Smith $\mathrm{CR}$, et al. 30-day readmissions after coronary artery bypass graft surgery in New York State. JACC Cardiovasc Interv 2011;4:569-76. Crossref

7. Hannan EL, Zhong Y, Krumholz H, Walford G, Holmes DR Jr, Stamato NJ, et al. 30-day readmission for patients undergoing percutaneous coronary interventions in New York state. JACC Cardiovasc Interv 2011;4:1335-42. Crossref

8. Khawaja FJ, Shah ND, Lennon RJ, Slusser JP, Alkatib AA, Rihal CS, et al. Factors associated with 30-day readmission rates after percutaneous coronary intervention. Arch Intern Med 2012;172:112-7. Crossref

9. Jennings DL, Petricca JC, Yageman LA, O’Dell K, Kalus JS. Predictors of rehospitalization after acute coronary syndromes. Am J Health Syst Pharm 2006;63:367-72. Crossref

10. Steuer J, Blomqvist P, Granath F, Rydh B, Ekbom A, de Faire U, et al. Hospital readmission after coronary artery bypass grafting: are women doing worse? Ann Thorac Surg 2002;73:1380-6.

11. Harjai KJ, Singh M, Boura J. Early readmissions after percutaneous coronary intervention in a rural tertiary care center (from the Guthrie Health Off-label Stent [GHOST] Registry). Am J Cardiol 2012;110:491-7. Crossref 